\title{
Scaling symmetry and conserved charge for shape-invariant optical fields
}

\author{
Omar El Gawhary* \\ VSL Dutch Metrology Institute, Thijsseweg 112629 JA Delft, Netherlands and \\ Optics Research Group, Department of Imaging and Technology, Delft University of Technology, \\ Van der Waalsweg 82628 CH Delft, Netherlands \\ Sergio Severini \\ Centro Interforze Studi per le Applicazioni Militari Via Bigattiera (lato monte) 10, 56122 San Piero a Grado (Pi), Italy
}

(Received 9 October 2012; published 13 February 2013)

\begin{abstract}
In this work we present an extensive study of the scaling symmetry typical of a paraxial wave theory. In particular, by means of a Lagrangian approach we derive the conservation law and the corresponding generalized charge associated with the scale invariance symmetry. In general, such a conserved charge, $q_{s}$ say, can take any value that remains constant during propagation. However, it is explicitly proven that for the whole class of physically realizable shape-invariant fields, that is, fields whose intensity distribution maintains its shape on propagation, $q_{s}$ must necessarily vanish. Finally, an interesting relation between such charge $q_{s}$ and the effective radius of a beam, as introduced by Siegman some years ago, is derived.
\end{abstract}

DOI: 10.1103/PhysRevA.87.023811

PACS number(s): 42.25.-p, 11.10.Ef, 11.30.-j

\section{INTRODUCTION}

Symmetries have always attracted a lot of interest from physicists, mainly because of their close connection with the fundamental properties of a system, as stated by Noether's theorem [1,2]. This is true in optics a well, where, in the last years, there was a notable growth in publication of works that studied and explained some of the features of optical fields by using symmetry arguments [3-8]. As an example, we can recall here the beautiful derivation, due to Wünche [5], of Hermite-Gauss and Laguerre-Gauss beams by application of certain differential operators to the fundamental Gaussian beam, introduced in the context of Lie's algebra for the paraxial regime. Another useful application of group theory to optics is the demonstration of the existence of the generic class of Gaussianly modulated paraxial beams obtained from the corresponding nondiffracting beams, solutions of Helmholtz's wave equation [6]. In another work, Simon and Mukunda [8] described in a universal way the propagation of shape-invariant fields by using the Iwasawa decomposition for the first-order optics. The goal of the present work is to present an extensive study of the scale-invariance symmetry in paraxial optics. This is performed by employing a Lagrangian description of a paraxial wave theory. Through our study, we will be able to point out the structure of the conservation law associated with the most general form of scaling symmetry and, more importantly, we will be able to derive a very general expression for the corresponding conserved charge $q_{s}$. Among all possible solutions of the paraxial wave equation, we apply the main results of our work to the interesting class of so-called shape-invariant fields, namely fields whose intensity profile does not change in propagation apart from a trivial scaling factor. We explicitly show that any shape-invariant field must necessarily bring a conserved charge $q_{s}=0$. Finally, we provide the reader with an interesting connection between the said charge $q_{s}$ and the concept, introduced several years

*oelgawhary@vsl.nl ago, of the average radius of curvature for a beam. The paper is organized as follows. In Sec. II we recall briefly the main ingredients of a Lagrangian theory, such as the Lagrangian and Lagrangian density, the energy-momentum tensor, and some related quantities. In Sec. III we analyze the scale invariance symmetry, by making use of some results of Lie's group theory applied to the paraxial wave equation and of Noether's theorem for scalar fields. Then we point out the explicit expression for the conserved scale-invariant current and charge. In Sec. IV we compute the value of the conserved charge associated with the scale-invariance symmetry for the case of shape-invariant fields and for the nontrivial case of a quartic-aberrated beam. Finally, in the last Sec. VI we summarize the results of our work.

\section{PARAXIAL OPTICS AS A FIELD THEORY}

From now on, we refer in the text to a $(x, y, z)$ Cartesian reference frame and we consider only monochromatic scalar paraxial fields, with the $z$ axis denoting the mean direction of propagation of the beam. It is known that a paraxial field $u(x, y, z)$, and its complex conjugate version $u^{\star}(x, y, z)$, must fulfill the wave equations

$$
\begin{aligned}
\nabla_{\perp}^{2} u(x, y, z)+2 i k \partial_{z} u(x, y, z) & =0, \\
\nabla_{\perp}^{2} u^{\star}(x, y, z)-2 i k \partial_{z} u^{\star}(x, y, z) & =0,
\end{aligned}
$$

that constitute the equations of motion for the fields in absence of sources and currents [9]. Equation (1a) is also known as paraxial wave equation (PWE). In Eqs. (1a) and (1b) $\nabla_{\perp}^{2}$ stands for the transverse Laplacian operator, which in the above-mentioned frame is given by $\nabla_{\perp}^{2}=\partial_{x}^{2}+\partial_{y}^{2}$. Since we intend to investigate the consequences deriving from the scaling symmetry of PWE, we need to recall briefly here the main features of such a symmetry. More specifically, a scaling symmetry of PWE is that symmetry transformation that, starting from a solution $u(x, y, z)$ for Eq. (1a), leads to the new solution

$$
\tilde{u}(x, y, z)=\exp (-\varepsilon) u[\exp (\varepsilon) x, \exp (\varepsilon) y, \exp (2 \varepsilon) z],
$$


where $\varepsilon$ is a real, positive parameter. Before analyzing the conserved quantities associated with this symmetry, we need to recall some preliminary facts on the Lagrangian formalism for field theories. For this reason, in the next sections we introduce a Lagrangian density and the canonical energy-momentum tensor associated with the PWE in Eq. (1a) [and its complex conjugated version, Eq. (1b)], pointing out some known conservation laws.

\section{A. The Lagrangian density $L_{D}$}

In any classical field theory, one can define the Lagrangian $\mathrm{L}$ as

$$
\mathrm{L}=\int_{\Sigma} L_{D} d x d y
$$

where

$$
L_{D}=L_{D}\left(u, u^{\star}, \partial_{\mu} u, \partial_{\mu} u^{\star}\right)
$$

is called Lagrangian density, where $\mu=\{x, y, z\}$ and $\Sigma$ denotes the integration domain in the $(x, y)$ space. It is possible to show that $L_{D}$ governs the field dynamics [10]. In fact, once the functional action $S$ is defined as

$$
S=\int_{\tau} L_{D} d x d y d z
$$

one can derive the equations of motion, known as EulerLagrange equations, by looking for those fields which extremize $S$. The expressions for the Euler-Lagrange equations obtained this way are

$$
\begin{gathered}
\frac{\partial L_{D}}{\partial u}-\partial_{\mu} \frac{\partial L_{D}}{\partial\left(\partial_{\mu} u\right)}=0, \\
\frac{\partial L_{D}}{\partial u^{\star}}-\partial_{\mu} \frac{\partial L_{D}}{\partial\left(\partial_{\mu} u^{\star}\right)}=0 .
\end{gathered}
$$

In our case we need to solve the inverse problem, because we already know the equations of motion, namely Eqs. (1a) and (1b), and we need to derive an expression for the Lagrangian density $L_{D}$. It is easily shown that the Lagrangian density

$$
\begin{aligned}
L_{D}\left(u, u^{\star}, \partial_{\mu} u, \partial_{\mu} u^{\star}\right) & =\partial_{a} u \partial^{a} u^{\star}+i k u \partial_{z} u^{\star}-i k u^{\star} \partial_{z} u \\
& =\nabla_{\perp} u \nabla_{\perp} u^{\star}+i k u \partial_{z} u^{\star}-i k u^{\star} \partial_{z} u \\
& =\left|\nabla_{\perp} u\right|^{2}+i k u \partial_{z} u^{\star}-i k u^{\star} \partial_{z} u
\end{aligned}
$$

(with $a=\{x, y\}$ ) leads to the correct equations of motion, given in Eqs. (1), when substituted into the Eqs. (6). We recall also here that the Lagrangian density is not unique. In fact, a term of form $d F(u ; z) / d z$ can be added without altering the value of the action $S$ in Eq. (5). Since the physics remains unchanged, and we are working only with observables, we set it to $F=0$. Additionally, for the sake of clarity throughout our work we keep the distinction between covariant and contravariant in the notation, although the metric would make it possible to identify them.

Finding a Lagrangian density is the fundamental step for our end, because by means of it one can introduce several quantities which play an important role in symmetry problems, as we show in the next section.

\section{B. Energy-momentum tensor}

Once the form for Lagrangian density $L_{D}$ is given, we can compute the Hamiltonian as follows:

$$
\mathrm{H}=-\frac{\partial L_{D}}{\partial\left(\partial_{z} u\right)} \partial_{z} u-\frac{\partial L_{D}}{\partial\left(\partial_{z} u^{\star}\right)} \partial_{z} u^{\star}+L_{D},
$$

which results to be

$$
\mathrm{H}=\partial_{a} u \partial^{a} u^{\star}=\left|\nabla_{\perp} u\right|^{2} .
$$

The integration of Eq. (9) produces a quantity, which we call total Hamiltonian energy, equal to

$$
\mathrm{E}=\int_{\Sigma} \mathrm{H} d x d y=\int_{\Sigma}\left|\nabla_{\perp} u\right|^{2} d x d y .
$$

In Appendix A we show how such Hamiltonian energy is related to the field energy as usually defined in the literature. One should also notice that Eq. (9) is a positive definite quantity, as it should be. Since the total Hamiltonian energy is conserved during free-space propagation, we expect that existence of an energy current, say $\mathbf{s}$, which satisfies the following continuity relation:

$$
\partial_{z} \mathrm{H}+\nabla_{\perp} \cdot \mathbf{s}=0 .
$$

In effect, if we define the current $\mathbf{s}$ as

$$
s^{a}=-\frac{\partial L_{D}}{\partial\left(\partial_{a} u\right)} \partial_{z} u-\frac{\partial L_{D}}{\partial\left(\partial_{a} u^{\star}\right)} \partial_{z} u^{\star},
$$

by using Eq. (7), it becomes

$$
s^{a}=-\partial^{a} u^{\star} \partial_{z} u-\partial^{a} u \partial_{z} u^{\star}
$$

and it is simple to check that $\mathbf{s}$ satisfies Eq. (11). More generally, it is straightforward to realize that the Hamiltonian $\mathrm{H}$ and the energy current $\mathbf{s}$ coincide, respectively, with the $T^{z z}$ and the $T^{z a}$ components of the following quantity (which is reminiscent of the energy-momentum tensor for classical field theories):

$$
T^{\alpha \beta}=-\frac{\partial L_{D}}{\partial\left(\partial_{\beta} u\right)} \partial^{\alpha} u-\frac{\partial L_{D}}{\partial\left(\partial_{\beta} u^{\star}\right)} \partial^{\alpha} u^{\star}+\eta^{\alpha \beta} L_{D},
$$

where $\eta^{\alpha \beta}=\delta^{\alpha \beta}$ and $\delta$ is the Kronecher symbol. The elements $T^{z z}$ and $T^{z a}$ have been already discussed above in the text. As to the remaining two other elements of Eq. (14), after denoting with $p^{a}$ the coefficient $T^{a z}$, we have

$$
\begin{aligned}
p^{a}=T^{a z} & =-\frac{\partial L_{D}}{\partial\left(\partial_{z} u\right)} \partial^{a} u-\frac{\partial L_{D}}{\partial\left(\partial_{z} u^{\star}\right)} \partial^{a} u^{\star} \\
& =i k u^{\star} \partial_{a} u-i k u \partial_{a} u^{\star}
\end{aligned}
$$

and

$$
T^{a b}=-\frac{\partial L}{\partial\left(\partial_{b} u\right)} \partial^{a} u-\frac{\partial L}{\partial\left(\partial_{b} u^{\star}\right)} \partial^{a} u^{\star}+\eta^{a b} L .
$$

One should notice that the tensor $T^{\alpha \beta}$ is not symmetric, but it is invariant under complex conjugation, i.e., $T^{\alpha \beta}=\left(T^{\alpha \beta}\right)^{\star}$. A very important relation fulfilled by $T^{\alpha \beta}$ is the following:

$$
\partial_{\beta} T^{\alpha \beta}=0,
$$

which can be proven to derive directly from the invariance of the theory under spatial translations. Equation (17) includes, 
as special cases, the energy conservation, already written in Eq. (11), and also the relation

$$
\partial_{z} T^{a z}+\partial_{a} T^{a b}=0
$$

between the vector $\mathbf{p}$ and the components $T^{a b}$. Before closing the present section we wish to emphasize that the vector $\mathbf{p}$ satisfies another continuity relation:

$$
\partial_{a} p^{a}-2 k^{2} \partial_{z}|u|^{2}=\nabla_{\perp} \cdot \mathbf{p}-2 k^{2} \partial_{z}|u|^{2}=0,
$$

which represents the well-known continuity equation for paraxial optics. With all the quantities defined so far, in the next section the focus is on the scale-invariance symmetry.

\section{CONSERVED CHARGE FOR SCALE-INVARIANCE SYMMETRY}

The most general form for a conservation law can be written as follows:

$$
\frac{1}{\sqrt{g}} \partial_{\mu}\left(\sqrt{g} J^{\mu}\right)=0
$$

where $\mathbf{J}$ represents a conserved current and $g$ the determinant of the metric tensor $g^{\alpha \beta}$. In the simplest cases $\sqrt{g}$ does not depend on $x, y, z$ variables so that Eq. (20) can be rewritten as

$$
\partial_{\mu} J^{\mu}=0 .
$$

If $u$ indicates a solution of Eq. (1a), we are interested here in the action of transformations that produce a scaling on spatial coordinates as well as on the field itself. For this purpose, it is convenient to associate with Eq. (1a) a manifold $M \in$ $X \times U$, where $X=\operatorname{Re}^{p}$, with $p=3$ meaning the number of independent variables and $U=\operatorname{Re}^{q}$, with $q=1$, meaning the number of dependent variables. A solution of PWE is represented on $M$ by a point $(x, y, z, u)$ while a map represents a symmetry transformation if it connects two different points, $(x, y, z, u)$ and $(\tilde{x}, \tilde{y}, \tilde{z}, \tilde{u})$, lying on the said manifold $M$. An application of Lie's group theory to the PWE allows us to obtain all the infinitesimal symmetry vector generators, among which there are $[6,11]$ :

$$
\begin{aligned}
& \mathbf{v}_{\mathbf{1}}=x \partial_{x}+y \partial_{y}+2 z \partial_{z}, \\
& \mathbf{v}_{\mathbf{2}}=u \partial_{u},
\end{aligned}
$$

which generate the maps

$$
\begin{aligned}
& \psi_{1}\left(\varepsilon, \mathbf{v}_{\mathbf{1}}\right)=\exp \left(\varepsilon \mathbf{v}_{\mathbf{1}}\right), \\
& \psi_{2}\left(\varepsilon, \mathbf{v}_{\mathbf{2}}\right)=\exp \left(\varepsilon \mathbf{v}_{\mathbf{2}}\right),
\end{aligned}
$$

where $\varepsilon$ is a real parameter characterizing the transformation. More specifically, Eq. (23) acts on the spatial variables to produce new solutions of the PWE, $u_{1}$ and $u_{2}$ say, as in the following:

$$
\begin{aligned}
& u_{1}(x, y, z)=u[\exp (\varepsilon) x, \exp (\varepsilon) y, \exp (2 \varepsilon) z], \\
& u_{2}(x, y, z)=\exp (-\varepsilon) u(x, y, z) .
\end{aligned}
$$

A transformation like that in Eq. (24b) is often called internal symmetry, because it involves only the field amplitude while leaving the independent variables $(x, y, z)$ undisturbed. To cite an example of an internal symmetry, we recall the action of a gauge transformation on the wave function in quantum mechanics [1,12]. On coming back to Eqs. (24), they can be combined to give the total scale-invariance transformation already written in Eq. (2). A physical system that shows a specific symmetry can have a conserved quantity associated with that symmetry. This can be formally stated by means of Noether's theorem (the reader interested in the details of the theorem might consider to look, for instance, at Ref. [1] for more details), which gives a way to build a conserved current $J^{\mu}$ as follows:

$$
J^{\mu}=v_{1}^{\nu} T_{\nu}^{\mu}+\left.\frac{\partial L}{\partial\left(\partial_{\mu} u\right)} \frac{d u_{2}}{d \varepsilon}\right|_{\varepsilon=0}+\left(\left.\frac{\partial L}{\partial\left(\partial_{\mu} u\right)} \frac{d u_{2}}{d \varepsilon}\right|_{\varepsilon=0}\right)^{\star},
$$

where $v_{1}^{v}$ are the components of the vector appearing into the transformations (22). In Eq. (25) there are two main terms:

(i) $v_{1}^{v} T_{v}^{\mu}$, which accounts for the action on the spatial coordinates $(x, y, z)$ only;

(ii) the term

$$
\left.\frac{\partial L}{\partial\left(\partial_{\mu} u\right)} \frac{d u_{2}}{d \varepsilon}\right|_{\varepsilon=0},
$$

which is due to the contribution of the internal symmetry Eq. (24b).

Furthermore, as we require the current to be observable, we have added the conjugate term of the internal symmetry, i.e., the term

$$
\left(\left.\frac{\partial L}{\partial\left(\partial_{\mu} u\right)} \frac{d u_{2}}{d \varepsilon}\right|_{\varepsilon=0}\right)^{\star} .
$$

After doing the calculations, the current $J^{\mu}$ assumes the final form

$$
J^{\mu}=v_{1}^{\alpha} T_{\alpha}^{\mu}+\frac{\partial L}{\partial\left(\partial_{\mu} u\right)}(-u)+\frac{\partial L}{\partial\left(\partial_{\mu} u^{\star}\right)}\left(-u^{\star}\right) .
$$

One can verify that this current is conserved, namely that $\partial_{\mu} J^{\mu}=0$. For the reader interested in the details, we have provided a proof of that in Appendix B.

To obtain the expression for the conserved charge associated to the conserved current, it is necessary to evaluate the integral

$$
\int_{\tau} \partial_{\mu} J^{\mu} d \tau=0
$$

where $\tau$ is a generic volume in $(x, y, z)$ space. More specifically, if we choose $\tau$ as the internal volume of a cylinder with symmetry axis parallel to the $z$ axis and with mantle $\Sigma_{m}$ and bases $\Sigma_{a}$ and $\Sigma_{b}$, Eq. (29) becomes, after applying the divergence theorem,

$$
\int_{\Sigma_{m}} \mathbf{J}_{\perp} \cdot d \mathbf{S}+\int_{\Sigma_{a}} J^{z} d S+\int_{\Sigma_{b}} J^{z} d S=0
$$

At this point we require the transverse current to vanish, i.e., $\mathbf{J}_{\perp} \rightarrow 0$, when the base radius of the cylinder becomes infinitely large. From that follows that the quantity

$$
q_{s}=\iint_{\Sigma_{\infty}} J^{z} d x d y
$$

is conserved during propagation. $q_{s}$ in Eq. (31) is the conserved charge we were looking for. Its explicit expression, evaluated 
on a general $z$ plane, can be rewritten as

$$
\begin{aligned}
q_{s} & =\left[\iint_{\Sigma_{\infty}}\left(x T_{x}^{z}+y T_{y}^{z}+2 z T_{z}^{z}\right) d x d y\right]_{z} \\
& =\left[\iint_{\Sigma_{\infty}}\left(x^{a} T_{a}^{z}+2 z T_{z}^{z}\right) d x d y\right]_{z} \\
& =\left[\iint_{\Sigma_{\infty}}\left(\mathbf{r}_{\perp} \cdot \mathbf{p}+2 z \mathrm{H}\right) d x d y\right]_{z} \\
& =\left[\iint_{\Sigma_{\infty}}\left(\mathbf{r}_{\perp} \cdot \mathbf{p}\right) d x d y\right]_{z}+2 z \mathrm{E},
\end{aligned}
$$

where $\mathrm{H}$ is the Hamiltonian. In Eq. (9), p is the linear momentum given in Eq. (15), $\mathbf{r}_{\perp}=(x, y)$ the transverse position vector, and $E$ the field energy already introduced in Eq. (10). The subscript $z$ means that all the quantities appearing within the square brackets have to be evaluated on a generic $z$ plane. The current density in Eq. (25) and the charge in Eq. (32) are the results which have motivated our investigation. In particular, from a different perspective, we can say that the scale-invariance symmetry of paraxial optics is induced by the conservation of the charge $q_{s}$ given in Eq. (32). In the next section we derive an important theorem for the value of $q_{s}$ in the case of important class of shape-invariant fields.

It is not straightforward to provide the reader with a physical meaning of the conserved charge $q_{s}$. From Eq. (32) we see that it depends on the integral of the scalar product $\left(\mathbf{r}_{\perp} \cdot \mathbf{p}\right)$. The field $\mathbf{p}$ is a linear momentum on the $(x, y)$ plane and it accounts for the way the energy flows on each transverse plane. Since the field keeps its energy during free-space propagation, it is evident that the transverse energy flow must be in balance with the total energy carried by the field, which is contained in the additional term $2 z \mathrm{E}$ in Eq. (32).

\section{THE VALUE OF $q_{s}$ FOR SHAPE-INVARIANT FIELDS}

Shape-invariant fields are special solutions of PWE sharing the property of having an intensity profile that remains essentially the same during propagation, apart from a scaling in the transverse coordinates. Gaussian, Hermite-Gauss, LaguerreGauss, and paraxial nondiffracting beams (Bessel, Mathieu beams) are some representatives of them [7,13-16]. In general, we can write their intensity as

$$
I\left(\mathbf{r}_{\perp}, z\right)=\alpha(z) I_{0}\left[\alpha(z) \mathbf{r}_{\perp}, 0\right],
$$

where $I=|u|^{2}$; the subscript denotes the $z=0$ plane. As to the function $\alpha(z)$ appearing in Eq. (33), it was elsewhere proven that

$$
\alpha(z)=\frac{1}{\sqrt{1 \pm\left(z / z_{R}\right)^{2}}},
$$

with $z_{R}$ as a real parameter [17]. It is interesting to notice that from Eqs. (19) and (33) already follows that, on $z=0$, $\nabla_{\perp} \cdot \mathbf{p}=0$. Hence, since $\mathbf{p}$ is free of divergence on $z=0$, it can be written as $\mathbf{p}=\nabla_{\perp} \times \mathbf{W}$, where $\mathbf{W}$ is a not further specified as a vector field. Thus, we can rewrite the integrand appearing in Eq. (32) as

$$
\begin{aligned}
\mathbf{r}_{\perp} \cdot \mathbf{p} & =\mathbf{r}_{\perp} \cdot \nabla_{\perp} \times \mathbf{W}=\nabla_{\perp} \cdot\left(\mathbf{r}_{\perp} \times \mathbf{W}\right)+\mathbf{W} \cdot \nabla_{\perp} \times \mathbf{r}_{\perp} \\
& =\nabla_{\perp} \cdot\left(\mathbf{r}_{\perp} \times \mathbf{W}\right),
\end{aligned}
$$

where we used the vector identity $\mathbf{B} \cdot \nabla \times \mathbf{A}=\nabla_{\perp} \cdot(\mathbf{A} \times$ $\mathbf{B})+\mathbf{A} \cdot \nabla_{\perp} \times \mathbf{B}$ and the fact that $\nabla_{\perp} \times \mathbf{r}_{\perp}=0$. Since the charge $q_{s}$ is not a function of the $z$ variable, it is sufficient to evaluate it on $z=0$ only. In that case, we have

$$
\begin{aligned}
q_{s}^{z=0} & =\left[\int_{\Sigma_{\infty}}\left(\mathbf{r}_{\perp} \cdot \mathbf{p}\right) d x d y\right]_{z=0} \\
& =\left[\int_{\Sigma_{\infty}} \nabla_{\perp} \cdot\left(\mathbf{r}_{\perp} \times \mathbf{W}\right) d x d y\right]_{z=0} \\
& =\left[\int_{\partial \Sigma_{\infty}} \mathbf{r}_{\perp} \times \mathbf{W} \cdot d \mathbf{l}\right]_{z=0},
\end{aligned}
$$

where a two-dimensional version of Gauss' theorem has been used. In Eq. (36) $\partial \Sigma_{\infty}$ is the border of the surface $\Sigma_{\infty}$ and $d \mathbf{l}$ represents the vector line element on such border. At this point, we further require to deal only with physically realizable fields, which implies that $\mathbf{W} \rightarrow 0$ for $\mathbf{r}_{\perp} \rightarrow \infty$. From Eq. (36), it is evident that this directly implies that $q_{s}=0$. Thus, we are led to conclude that $q_{s}=0$ for any physically realizable shape-invariant field.

The converse, however, is not true. In other words, it is possible to have fields with $q_{s}=0$ which are not shape invariant. This can be seen by rewriting the charge $q_{s}$ in a different way. In particular, if we write the complex field $u$ in terms of its amplitude and phase as $u(x, y, z)=$ $[I(x, y, z)]^{1 / 2} \exp [i \varphi(x, y, z)]$, it follows that

$$
\mathbf{p}=i k u^{\star} \nabla_{\perp} u-i k u \nabla_{\perp} u^{\star}=-2 k I \nabla_{\perp} \varphi
$$

and for $q_{s}$ we get

$$
\begin{aligned}
q_{s} & =\left[\iint_{\Sigma_{\infty}}-2 k I\left(r_{\perp} \cdot \nabla_{\perp} \varphi\right) d x d y\right]_{z}+2 z \mathrm{E} \\
& =\left[\int_{0}^{2 \pi} \int_{0}^{\infty}-2 k I r_{\perp}^{2} \partial_{r_{\perp}} \varphi d r d \theta\right]_{z}+2 z \mathrm{E},
\end{aligned}
$$

where the standard relations $x=r_{\perp} \cos \theta, y=r_{\perp} \sin \theta$ between Cartesian and circular coordinates have been used. On $z=0$, Eq. (38) becomes

$$
q_{s}=\left[\int_{0}^{2 \pi} \int_{0}^{\infty}-2 k I r_{\perp}^{2} \partial_{r_{\perp}} \varphi d r_{\perp} d \theta\right]_{z=0} .
$$

Equation (39) points out how the phase of the field, on the input plane, can affect the conserved charge $q_{s}$. It is interesting to discuss some particular cases. Let us consider first a field with a phase that, on $z=0$, does not depend on $r_{\perp}$. In such a case we have $\partial_{r_{\perp}} \varphi=0$ and $q_{s}$ vanishes. In other words, given a field $u(x, y, z)=[I(x, y, z)]^{1 / 2} \exp [i \varphi(x, y, z)]$, then $a$ sufficient condition to have a vanishing conserved charge $q_{s}$ is $\left.\partial_{r_{\perp}} \varphi\right|_{z=0}=0$. The condition is sufficient but not necessary because, as is easy to show, $q_{s}$ can be zero also when $\left.\partial_{r_{\perp}} \varphi\right|_{z=0} \neq 0$. For instance, let us consider the case of a field of type $u(x, y, 0)=u\left(r_{\perp}, \theta, 0\right)=\left[I\left(r_{\perp}\right)\right]^{1 / 2} \exp \left[i \varphi\left(r_{\perp}, \theta\right)\right]$ with a phase of form $\varphi\left(r_{\perp}, \theta\right)=f\left(r_{\perp}\right) g(\theta)$, where $f\left(r_{\perp}\right)$ and $g(\theta)$ are two functions of $r_{\perp}$ and $\theta$ variables, respectively. In that case $q_{s}$ becomes

$$
q_{s}=\left[\int_{0}^{2 \pi} g(\theta) d \theta\right]\left[\int_{0}^{\infty}-2 k I r_{\perp}^{2} \partial_{r_{\perp}} f\left(r_{\perp}\right) d r_{\perp}\right]_{z=0}=0
$$


because $\int_{0}^{2 \pi} g(\theta) d \theta=0$, since the function $g(\theta)$ has to be periodic on $\theta$.

As an example of a field that has a conserved charge $q_{s}$ different from zero, let us consider the case of a quarticaberrated Gaussian beam that, on the plane $z=0$, takes the expression

$$
u(x, y, 0)=A \exp \left(-\frac{r_{\perp}^{2}}{w_{0}^{2}}+i \beta r_{\perp}^{4}\right),
$$

where $\beta$ describes the said aberration and $w_{0}$ represents the well-known beam waist of a nonaberrated Gaussian beam. In this case one has $\left.\partial_{r_{\perp}} \varphi\right|_{z=0}=3 \beta r^{3}$ and the conserved charges result to be depending on the introduced aberration

$$
\begin{aligned}
q_{s}^{(0)} & =\int_{0}^{2 \pi} \int_{0}^{\infty}-2 k A^{2} \exp \left(-\frac{2 r_{\perp}^{2}}{w_{0}^{2}}\right) r_{\perp}^{2} 3 \beta r_{\perp}^{3} d r_{\perp} d \theta \\
& =-12 \beta \pi k \int_{0}^{\infty} A^{2} \exp \left(-\frac{2 r_{\perp}^{2}}{w_{0}^{2}}\right) r_{\perp}^{5} d r_{\perp} \\
& =-\frac{3}{2} \beta \pi k w_{0}^{6}
\end{aligned}
$$

which is different from zero and directly proportional to the aberration parameter $\beta$.

\section{RELATIONSHIP BETWEEN THE CONSERVED CHARGE $\boldsymbol{q}_{s}$ AND THE EFFECTIVE RADIUS OF CURVATURE OF A BEAM}

There exists an interesting connection between the justintroduced conserved charge $q_{s}$ and the effective radius of curvature of a general paraxial beam as defined, several years ago, by Siegman [18]. Such a quantity was introduced to describe the effective radius of curvature of a real beam even when it may be characterized by a distorted wave front and by a very irregular amplitude profile. For the sake of simplicity, we refer to an axially symmetric beam, for which an average curvature radius can be defined, on $z$, as

$$
R_{z}=-k \sigma_{z}^{2}\left[2 \pi \int_{0}^{\infty} r_{\perp}^{2} \partial_{r} \varphi I d r_{\perp}\right]_{z}^{-1},
$$

where $\sigma_{z}^{2}$ is the variance of the transverse intensity distribution, propagating on $z$ according to a quadratic law

$$
\sigma_{z}^{2}=\sigma_{0}^{2}\left[1+\left(\frac{M^{2} z}{k \sigma_{0}^{2}}\right)^{2}\right] \text {. }
$$

Here $\sigma_{0}$ is the value of $\sigma_{z}$ evaluated on $z=0$. As is well known, the $M^{2}$ accounts for spreading properties of the beam during free-space propagation. The said relation between $R_{z}$ and $q_{s}$ readily follows after comparing (38) and (44) and reads

$$
R_{z}=-k \sigma_{0}^{2} \frac{\left[1+\left(\frac{M^{2} z}{k \sigma_{0}^{2}}\right)^{2}\right]}{q_{s}-2 z \mathrm{E}} .
$$

Equation (46) represents a generalization of the result given by Siegman in his already cited work [18]. In fact, Siegman introduced a propagation law for the average radius of curvature $R_{z}$, for a generic paraxial beams, that can be simply obtained by putting $q_{s}=0$ into Eq. (46). Thus, Eq. (46) in some sense is a generalization of that propagation law to cases where $q_{s} \neq 0$. More interestingly, apart from the dependence on $z$, Eq. (46) clearly shows that $R_{z}$ is exclusively expressed as a function of the field invariants $q_{s}, \sigma_{0}, M^{2}$, E.

\section{CONCLUSIONS}

In the present work we have performed a detailed study about the scale-invariance symmetry in paraxial optics. By using a Lagrangian description for the paraxial regime, we have obtained the generic structure of the conserved current and charge $q_{s}$ associated with the said symmetry. Additionally, we have shown that shape-invariant fields have always $q_{s}=0$. We have discussed how such a conserved charge is influenced by the behavior of the phase of a field under study, in particular by the phase profile on an input plane. Conditions to obtain a nonvanishing charge $q_{s}$ have been discussed as well. In addition, we have shown how the concept of average radius of curvature of a generic beam actually is founded on the scale-invariance properties of paraxial optical beams.

\section{ACKNOWLEDGMENTS}

The authors are grateful to Amalia Torre for useful comments about the subject of the present paper, as well as for suggesting some improvements.

\section{APPENDIX A: RELATIONSHIP BETWEEN THE HAMILTONIAN ENERGY AND FIELD ENERGY}

In Sec. II A we recalled how, within a Lagrangian picture, it is possible to define a conserved energy $\mathrm{E}$, which is the total Hamiltonian energy. At a first sight, this energy seems to differ from the usual way the energy of a paraxial beam is defined. The aim of the present section is to show how the two definitions of energy are actually related to each other.

If $u(x, y, z)$ denotes the field under consideration, then we can define its energy in the usual way as the integral $\int|u|^{2} d x d y$. If we now sum Eqs. (11) and (19) together we obtain

$$
\nabla_{\perp} \cdot(\mathbf{p}+\mathbf{s})+\partial_{z}\left(\mathrm{H}-2 k^{2}|u|^{2}\right)=0 .
$$

Integrating over the whole $x, y$ plane and requiring that both $\mathbf{p}$ and $\mathbf{s}$ must vanish at infinity, we get

$$
\partial_{z} \int_{\Sigma_{\infty}}\left(\mathrm{H}-2 k^{2}|u|^{2}\right) d x d y=0,
$$

where we made use of Gauss's theorem. Equation (A2) implies that the integral

$$
\int_{\Sigma_{\infty}}\left(\mathrm{H}-2 k^{2}|u|^{2}\right) d x d y
$$

is invariant on propagation. Thus, we have

$$
\mathrm{E}=C+2 k^{2} \int_{\Sigma_{\infty}}|u|^{2} d x d y,
$$

where $C$ is just a constant term. Equation (A4) represents the relation we were looking for. By setting the constant $C$ to zero, one obtains the relevant result that, apart from 
a trivial proportional factor, E coincides with the field energy for paraxial fields, as commonly defined in the literature.

\section{APPENDIX B: PROOF OF THE CONSERVATION OF THE SCALE-INVARIANCE CURRENT}

In the present appendix we explicitly prove that the current given in Eq. (28), is conserved. We work in Cartesian coordinates.

We obtain for the $J^{x}$ and $J^{y}$ components

$$
\begin{aligned}
J^{x} & =v_{1}^{\alpha} T_{\alpha}^{x}+\frac{\partial L_{D}}{\partial\left(\partial_{x} u\right)}(-u)+\frac{\partial L_{D}}{\partial\left(\partial_{x} u^{\star}\right)}\left(-u^{\star}\right) \\
& =x T_{x}^{x}+y T_{y}^{x}+2 z T_{z}^{x}+\partial_{x} u^{\star}(-u)+\partial_{x} u\left(-u^{\star}\right), \\
J^{y} & =v_{1}^{\alpha} T_{\alpha}^{y}+\frac{\partial L_{D}}{\partial\left(\partial_{y} u\right)}(-u)+\frac{\partial L_{D}}{\partial\left(\partial_{y} u^{\star}\right)}\left(-u^{\star}\right) \\
& =x T_{x}^{y}+y T_{y}^{y}+2 z T_{z}^{y}+\partial_{y} u^{\star}(-u)+\partial_{y} u\left(-u^{\star}\right),
\end{aligned}
$$

while for $J^{z}$ it reads

$$
\begin{aligned}
J^{z} & =v_{1}^{\alpha} T_{\alpha}^{z}+\frac{\partial L_{D}}{\partial\left(\partial_{z} u\right)}(-u)+\frac{\partial L_{D}}{\partial\left(\partial_{z} u^{\star}\right)}\left(-u^{\star}\right) \\
& =x T_{x}^{z}+y T_{y}^{z}+2 z T_{z}^{z}+\left(-i k u^{\star}\right)(-u)+\left(+i k u^{\star}\right)\left(-u^{\star}\right) \\
& =x T_{x}^{z}+y T_{y}^{z}+2 z T_{z}^{z} .
\end{aligned}
$$

The divergence of $\mathbf{J}$ becomes

$$
\begin{aligned}
\partial_{\mu} J^{\mu}= & T_{x}^{x}+x \partial_{x} T_{x}^{x}+y \partial_{x} T_{y}^{x}+2 z \partial_{x} T_{z}^{x}-u \partial_{x}^{2} u^{\star}-\partial_{x} u^{\star} \partial_{x} u \\
& -u^{\star} \partial_{x}^{2} u-\partial_{x} u \partial_{x} u^{\star}+x \partial_{y} T_{x}^{y}+y \partial_{y} T_{y}^{y}+T_{y}^{y} \\
& +2 z T_{z}^{y}-u \partial_{y}^{2} u^{\star}-\partial_{y} u^{\star} \partial_{y} u-u^{\star} \partial_{y}^{2} u-\partial_{y} u \partial_{y} u^{\star} \\
& +x \partial_{z} T_{x}^{z}+y \partial_{z} T_{y}^{z}+2 z \partial_{z} T_{z}^{z}+2 T_{z}^{z} .
\end{aligned}
$$

Equation (B4) simplifies by using the conservation laws already discussed, in particular that in Eq. (17), and gives

$$
\begin{aligned}
\partial_{\mu} J^{\mu}= & x\left(\partial_{x} T_{x}^{x}+\partial_{y} T_{x}^{y}+\partial_{z} T_{x}^{z}\right)+y\left(\partial_{x} T_{y}^{x}+\partial_{y} T_{y}^{y}+\partial_{z} T_{y}^{z}\right) \\
& +2 z\left(\partial_{x} T_{z}^{x}+\partial_{y} T_{z}^{y}+\partial_{z} T_{z}^{z}\right)+T_{x}^{x}+T_{y}^{y}+2 T_{z}^{z} \\
& -u \partial_{x}^{2} u^{\star}-\partial_{x} u^{\star} \partial_{x} u-u^{\star} \partial_{x}^{2} u-\partial_{x} u \partial_{x} u^{\star}-u \partial_{y}^{2} u^{\star} \\
& -\partial_{y} u^{\star} \partial_{y} u-u^{\star} \partial_{y}^{2} u-\partial_{y} u \partial_{y} u^{\star} \\
= & T_{x}^{x}+T_{y}^{y}+2 T_{z}^{z}-u \partial_{x}^{2} u^{\star}-\partial_{x} u^{\star} \partial_{x} u-u^{\star} \partial_{x}^{2} u \\
& -\partial_{x} u \partial_{x} u^{\star}-u \partial_{y}^{2} u^{\star}-\partial_{y} u^{\star} \partial_{y} u-u^{\star} \partial_{y}^{2} u-\partial_{y} u \partial_{y} u^{\star} \\
= & 0,
\end{aligned}
$$

in which we have used the explicit form of $T_{x}^{x}, T_{y}^{y}$ (we have already pointed out that $T_{z}^{z}$ coincides with the Hamiltonian density $\mathrm{H})$, i.e.,

$$
\begin{array}{r}
T_{x}^{x}=-\partial_{x} u \partial_{x} u^{\star}+\partial_{y} u \partial_{y} u^{\star}+i k u \partial_{z} u^{\star}-i k u^{\star} \partial_{z} u, \\
T_{y}^{y}=-\partial_{y} u \partial_{y} u^{\star}+\partial_{x} u \partial_{x} u^{\star}+i k u \partial_{z} u^{\star}-i k u^{\star} \partial_{z} u,
\end{array}
$$

and we have used Eqs. (1), too.
[1] B. Felsager, Geometry, Particles, and Fields (Springer-Verlag, New York, 1998).

[2] C. Cohen-Tannoudji, J. Dupont-Roc, and G. Grynberg, Photons and Atoms (Wiley-VCH, Hoboken, NJ, 1997).

[3] O. El Gawhary and S. Severini, Opt. Commun. 283, 2481 (2010).

[4] O. El Gawhary and S. Severini, Opt. Lett. 33, 1360 (2008).

[5] A. Wünsche, J. Opt. Soc. Am. A 6, 1320 (1989).

[6] O. El Gawhary and S. Severini, J. Opt A: Pure Appl. Opt. 8, 409 (2006).

[7] J. C. Gutiérrez-Vega and M. A. Bandres, J. Opt. Soc. Am. A 22, 289 (2005).

[8] R. Simon and N. Mukunda, J. Opt. Soc. Am. A 15, 2146 (1998).

[9] M. Lax, W. H. Louisell, and W. B. McKnight, Phys. Rev. A 11, 1365 (1975).

[10] Throughout the paper, Greek letters indicate all coordinates, namely $\alpha, \beta, \gamma, \ldots=x, y, z$, while the lower-case letters such as $a, b, c, \ldots$ indicate only the transverse coordinates $x, y$.
[11] P. Olver, Applications of Lie Groups to differential equations, 2nd ed. (Springer-Verlag, New York, 1993).

[12] J. J. Sakurai, Modern Quantum Mechanics, revised ed. (Addison-Wesley, Boston, 1993).

[13] J. Durnin, J. J. Miceli, Jr., and J. H. Eberly, Phys. Rev. Lett. 58, 1499 (1987).

[14] C. J. R. Sheppard and T. Wilson, IEE J. Microwaves, Opt. Acoust. 2, 105 (1978).

[15] O. El Gawhary, S. F. Pereira, and H. P. Urbach, Phys. Rev. A 83, 033412 (2011).

[16] Q. Huang, S. Coetmellec, F. Duval, A. Louis, H. Leblond, and M. Brunel, J. Eur. Opt. Soc. Rapid Publ. 6, 11031 (2011).

[17] R. Borghi, M. Santarsiero, and R. Simon, J. Opt. Soc. Am. A 21, 572 (2004).

[18] A. E. Siegman, IEEE J. Quantum Electron. 27, 1146 (1991) 